\title{
A New Modelling and Feature Extraction Method Based on Complex Network and Its Application in Machine Fault Diagnosis
}

\author{
Qi Zhang, ${ }^{1}$ Tian Tian, ${ }^{1}$ Guangrui Wen $\mathbb{D}^{1,2,3}$ and Zhifen Zhang ${ }^{1}$ \\ ${ }^{1}$ School of Mechanical Engineering, Xi'an Jiaotong University, Xi'an, China \\ ${ }^{2}$ School of Mechanical Engineering, Xinjiang University, Wulumuqi, China \\ ${ }^{3}$ Key Laboratory of Education Ministry for Modern Design \& Rotor-Bearing System, Xi'an, China \\ Correspondence should be addressed to Guangrui Wen; grwen@mail.xjtu.edu.cn
}

Received 27 June 2018; Revised 9 September 2018; Accepted 2 October 2018; Published 2 December 2018

Academic Editor: Paolo Pennacchi

Copyright (c) 2018 Qi Zhang et al. This is an open access article distributed under the Creative Commons Attribution License, which permits unrestricted use, distribution, and reproduction in any medium, provided the original work is properly cited.

The application of the existing complex network in fault diagnosis is usually modelled based on the time domain, resulting in the loss of sign frequency-domain features, and the extracted topology features of network are too macroscopic and insensitive to local changes within the network. This paper proposes a new method of local feature extraction based on frequency complex network (FCN) decomposition and builds a new complex network structure feature on this basis, namely, subnetwork average degree. The variation law of signals in frequency domain is obtained with the aid of the structural features of complex network. The local features that are sensitive to local changes of the network are applied to characterize the whole network, with flexible application and without limitation in mechanism. The average degree of subnetwork could be regarded as feature parameters for rolling bearing fault diagnosis and degradation state recognition. Analysis on the experimental data and bearing life cycle data shows that the method proposed in this paper is effective, revealing that the extracted features have effective separability and high accuracy in fault recognition and the degradation detection of the life cycle of rolling bearings combined with neural networks. Moreover, the proposed method has reference value for the processing and recognition of other nonstationary signals.

\section{Introduction}

Rotating machinery is widely applied in large-scale petroleum, chemical, electric power, metallurgy, and other industries, serving as the core equipment. Its fault will not only affect the operation of the machine itself but also cause loss in follow-up production [1]. Therefore, it is of great practical significance to conduct fault diagnosis for rotary machinery. The nonstationary nature of vibration signals of a rotating machine often leads to difficulty in accurately identifying faults. Time-frequency analysis and wavelet, both effective methods to process nonlinear and nonstationary signals [2], still have different degrees of defects. For example, the selection of wavelet basis in wavelet analysis directly affects the analysis result, and the basis function, without adaptive feature, cannot be changed once selected in the analysis [3].

Complex network, a new method to analyze nonlinear and nonstationary signals, can transform the wave features of signals into the topological features of the network, which overcomes the shortcomings of traditional time-frequency analysis $[4,5]$. It is a simple, intuitive, universal, topological, and strong robust network structure [6,7]. Visible network construction method proposed by Lacasa and Toral can process time series of larger amount of data [8]. Ming et al. presented a new method to construct directed and weighted complex network based on time series symbolic pattern with sliding window method [9]. Recursive complex network was applied by Sun et al. to extract network features of rolling bearings and diagnose faults [10]. Although complex network has achieved good results in nonlinear and nonstationary signal analysis, current diagnostic methods of complex network is usually based directly on time domain, ignoring frequency-domain features, and the extracted features have only global features, without the reflection of local structures of networks, which is difficult to ensure the accurate extraction of fault information. 
Therefore, a new extraction method based on frequency complex network (FCN) model construction and subnetwork average degree is proposed in this paper and is applied in the bearing fault diagnosis, the monitoring and early warning of rolling bearings and the recognition of degradation state. The variation law of fault signal in frequency domain, obtained with the structural features of complex network, is transformed into the statistical features of the complex network structure, without limitation in fault mechanism. The verification results of fault signal of rolling bearings prove that this method is effective in extracting information that represents different fault features of the machine and can achieve accurate differentiation, providing a new effective approach for fault diagnosis of rotary machinery.

\section{Principle}

2.1. Recursive Algorithm. Based on its inherent characteristics, recursion can be used to show the similar properties in some specific states. The recursive graph principle proposed by Eckmann and others is applied in this paper to visualize recursive graphs using recursive matrixes to characterize the recursive nature of the system [11].

The recursive matrix uses phase space reconstruction to reconstruct a one-dimensional sequence into a high dimensional space. The specific algorithm is as follows:

(1) The power system reconstructed by one-dimensional series $x_{i}$ from the time delay $\tau$, and the embedded vector of the $m$-dimensional phase space is as follows:

$$
\begin{aligned}
x_{i}= & \left(u_{i}, u_{i+\tau}, \ldots, u_{i+(m-1) \tau}\right), \\
& i=1,2, \ldots, N-(m-1) \tau .
\end{aligned}
$$

(2) The distance between the reconstructed phase space $i$ and $j$ is calculated

$$
\begin{aligned}
S_{i j}=\left\|x_{i}-x_{j}\right\|, & i=1,2, \ldots, N-(m-1) \tau, \\
& j=1,2, \ldots, N-(m-1) \tau .
\end{aligned}
$$

(3) Recursive value is calculated

$$
\begin{aligned}
R(i, j)=H\left(\varepsilon-S_{i j}\right), & i=1,2, \ldots, N-(m-1) \tau, \\
j & =1,2, \ldots, N-(m-1) \tau,
\end{aligned}
$$

where $H(g)$ is Heaviside function, $\|g\|$ is Euclidean norm, and $\varepsilon$ is reference threshold value.

\subsection{Construction of Subnetwork Average Degree}

2.2.1. Subnetwork Average Degree. The topological features of networks include the average path length, clustering coefficient, degree and degree distribution, etc., among which degree is a simple yet important concept in the attributes of individual nodes.

$k_{i}$, the degree of a node, is defined as the number of other nodes connected to the node, which reflects the importance of this node. The bigger the degree of a node, the more important the node is [12]. The average degree of a complex network with the number of nodes $n$ is denoted as $p$ which is the mean of the degree of all nodes in the network $k_{i}$, i.e.,

$$
p=\frac{1}{n} \sum_{i=1}^{n} k_{i},
$$

where $n$ is the total number of nodes in the network.

The new complex network structure feature (subnetwork average degree) is extracted based on frequency complex network (FCN) decomposition, which reflects degree distribution of different regions in the network. The value of node degree in every region, the arrangement of node degrees, and the location of important node are all correlated. The network decomposition is to evenly divide the established complex network model into several subnetworks on the basis of the order of nodes (the edges of subnetworks are reserved during decomposing), and then, the average degree of each subnetwork can be obtained with formula (4) to generate average degree features of subnetworks, as shown in formula (5), namely,

$$
\vec{p}=\left[p_{1}, p_{2}, p_{3}, \ldots, p_{m}\right]
$$

wherein, when determining the number $m$ of subnetworks, $m=2^{l}$ is generally selected and should meet the requirement $2 \leq m \leq n$.

2.2.2. Analysis of the Advantages of Subnetwork Average Degree. Compared with the existing average degree features, the average degree of subnetworks can reflect the local features of the network in space with more information, which is more effective in fault recognition. The networks (a) and (b) in Figure 1, both six-node network models, have the same nodes with different connections between nodes. The number of connected edges of the nodes indicates that the important node of the network (a) is 2 and that of network (b) is 6 .

The degree of each node in the network needs to be calculated before the analysis on the degree of network node, with the calculation results shown in Table 1. If the network is characterized by the average value of degree of all nodes, the eigenvalues of the two networks are 2.67 with formula (4), hence no difference between the network (a) and the network (b).

However, if the average degree of subnetworks is extracted, the difference between the two networks can be reflected. The network is evenly divided into two subnetworks, with subnetwork 1 as the connection of nodes 1, 2, and 3 and subnetwork 2 as the connection of nodes 4,5 , and 6 . The average degree of subnetworks is generated and a twodimensional feature vector is constructed. Therefore, the feature vector of the network (a) is $[2.33,3]$ and that of the network (b) is $[3,2.33]$. It is not difficult to find out that the 

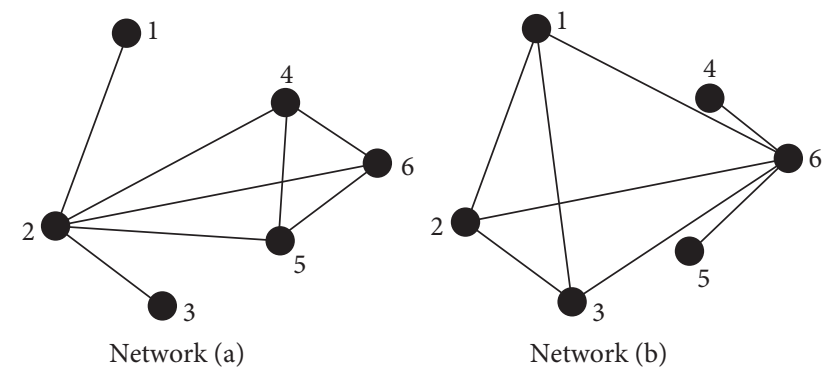

Figure 1: Six-node network model. Networks (a) and (b).

TABle 1: Network node degree.

\begin{tabular}{lllllll}
\hline Node & 1 & 2 & 3 & 4 & 5 & 6 \\
\hline Network (a) & 1 & 5 & 1 & 3 & 3 & 3 \\
Network (b) & 3 & 3 & 3 & 1 & 1 & 5 \\
\hline
\end{tabular}

average degree of subnetworks can mirror the difference between two networks in node connection, which can more accurately reflect the structural features of the network models.

\subsection{An Extraction Method Based on FCN Subnetwork Average} Degree. In view of the nonstationary nature of vibration signals of rotating machinery and the abundant information contained in frequency domain [13], a new subnetwork average degree feature is proposed in this paper based on frequency complex network (FCN) decomposition. The fault diagnosis process based on subnetwork average degree is shown in Figure 2.

The processing steps are as follows:

(1) Rotation frequency is calculated as $f_{\mathrm{r}}=V_{\mathrm{r}} / 60$. Frequency bandwidth $m$ is chosen as an integer multiple of $f_{\mathrm{r}}$ or $f_{\mathrm{r}}$. The frequency spectrum $Y=$ $\left\{y_{i}\right\}_{i=1,2, \ldots, n}$ is obtained from the one-dimensional time series $X$ using Fourier transform. The amplitudes are then squared point by point, added in the frequency band with bandwidth $m$ and arranged in order to obtain the required band energy spectrum [14], denoted as $E=\left[E_{1}, E_{2}, \ldots, E_{k}, \ldots, E_{n}\right]$. Wherein, $E_{k}$ represents the energy of the $K^{\text {th }}$ frequency band, with the formula

$$
E_{k}=\sum_{i=j}^{j+m} y_{i}^{2}
$$

(2) On the basis of the recursive algorithm, the recursive matrix $R$ reflecting self-similarity of energy spectrum can be obtained. The recursive matrix is regarded as an adjacency matrix to construct a complex network model. The average degree of subnetworks $p$ is extracted as the feature parameters with formula (4) and (5).

(3) In all samples, half of the data are randomly selected to form the training set, and the remaining half are test set. The radical basis function (RBF) neural

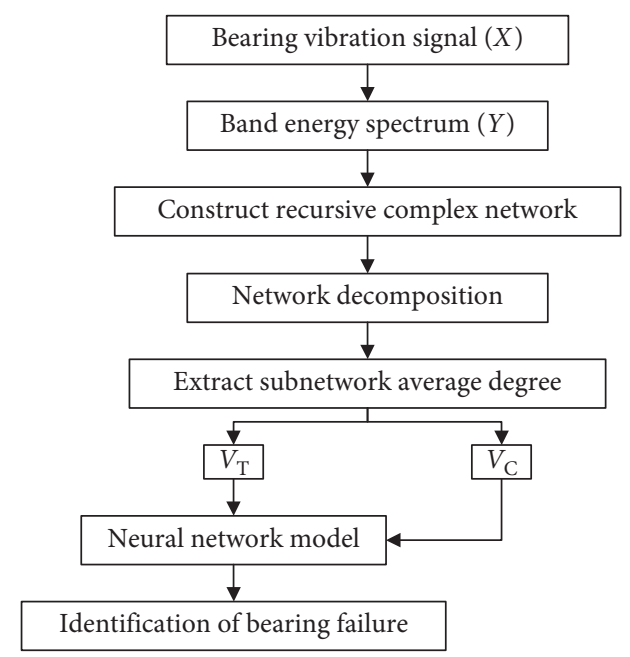

Figure 2: Flowchart for the bearing fault diagnosis based on subnetwork average degree.

network [15] is trained with the training sample feature vector set $V_{\mathrm{T}}$ to obtain the network model of the system. Then, the network is tested and verified with the testing sample feature vector set $V_{\mathrm{C}}$ to identify the types of the testing sample.

2.4. Performance Degradation Assessment Method Based on Network Structural Features. Targeting the problems of insensitivity of the traditional performance degradation index to early weak faults and the low accuracy of degradation state recognition [16], this paper proposes a new method to assess the bearing performance degradation based on the structural features of frequency complex network, with the processing flow shown in Figure 3.

The basic steps of this method are as follows:

(1) Extraction of performance degradation index: the bearing vibration signal is transformed into the frequency band energy spectrum, denoted as $E=\left[E_{1}, E_{2}, \ldots, E_{k}, \ldots, E_{n}\right]$, where $E_{k}$ represents the energy of the $k^{\text {th }}$ frequency band, with the formula being $E_{k}=\sum_{i=j}^{j+m} y_{i}^{2}(6)$. The recursive matrix $R$ is obtained according to the self-similarity of the energy spectrum in different frequency bands. The recursive matrix is regarded as an adjacency matrix to construct a complex network, and the network average degree $p$ is extracted as the bearing performance degradation index with formula (4).

(2) State monitoring: the Layda rule [17] is applied to determine the threshold on the basis of the average degree change curve. When the absolute value of the difference between the mathematical expectation of the data point and its set is greater than the standard deviation of the data set of 3 times, namely, $\left|x_{i}-\bar{x}\right|>3 \sigma$, the data point is determined as abnormal data. When the number of abnormal data consecutively exceeds the number of safety times, a fault alert is automatically generated. 


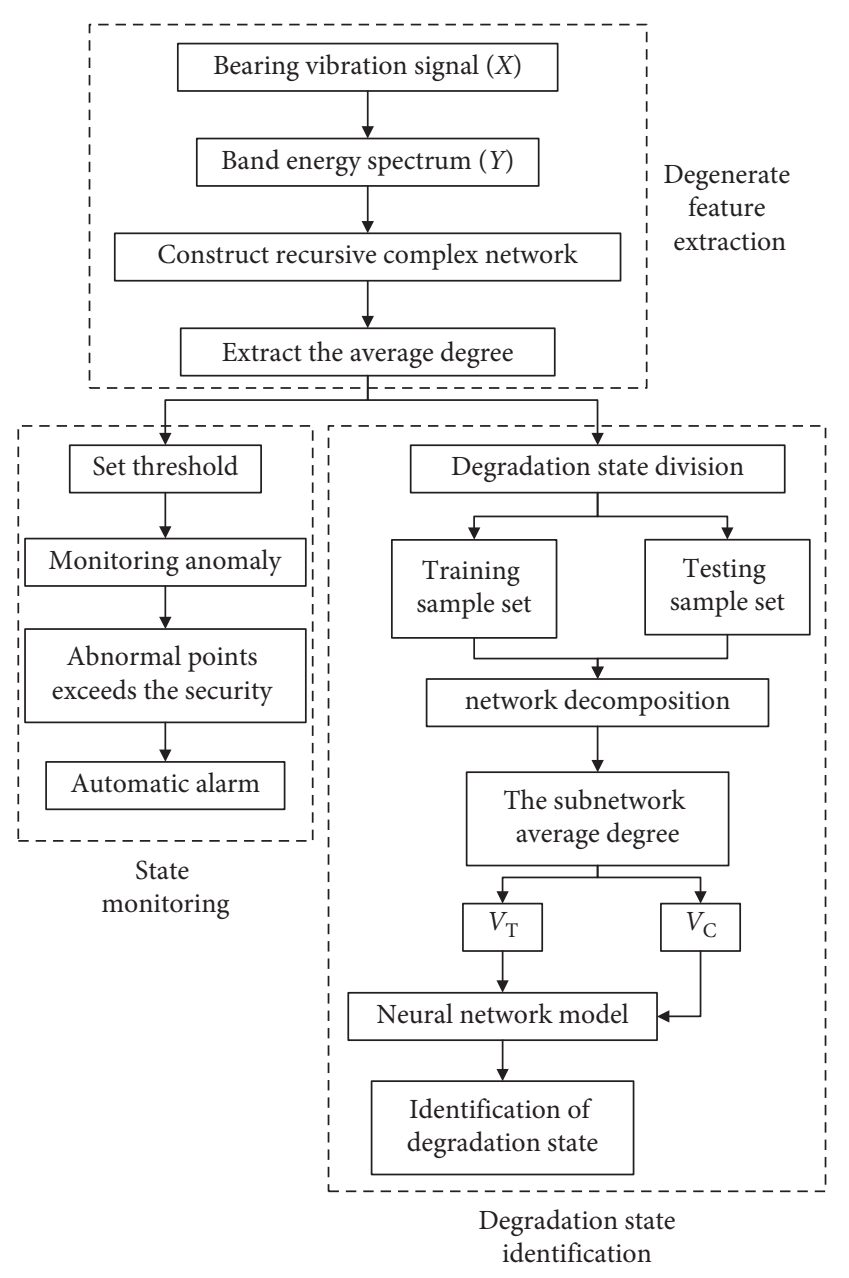

FIGURE 3: Flowchart for performance degradation assessment based on structural features from frequency complex network.

(3) Degradation state division: based on the evolution law of the performance degradation index $p$, the key data mutation points are determined. The degradation states of the bearing performance are approximately divided into four operating states: normal, minor fault, deepening of the fault and gradual thinning of the sharp part, and serious fault to failure.

(4) Determination of the training set and testing set: the samples in each degradation state are divided into two sample sets according to the parity of the serial number. One sample set is selected as the training sample set, and the other sample set is selected as the testing sample set.

(5) Extraction of multidimensional degradation feature vector: the complex network model corresponding to each sample is evenly decomposed. The number of subnetworks $m$ decomposed by the complex network is determined based on the total number of nodes in the complex network with the principle of $m=2^{l}$ and $2 \leq m \leq n$. Then, the average degree vector is extracted as $\vec{p}=\left[p_{1}, p_{2}, p_{3}, \ldots, p_{m}\right]$ as shown in formula (5). The degradation feature vector set of the training set $V_{\mathrm{T}}$ and the testing set $V_{\mathrm{C}}$ is then generated.

(6) Recognition of degradation state: the number of state categories is set as 4, and the RBF neural network is trained with the training sample feature vector set $V_{\mathrm{T}}$ to obtain the network model of the system. Then, the network is tested and verified with the testing sample feature vector set $V_{\mathrm{C}}$ to identify the degradation state of the testing sample.

\section{Data Validation and Analysis}

3.1. Extraction of FCN Subnetwork Average Degree of Bearing Condition. In this paper, the vibration signals of rolling bearings of different fault types are applied to verify the effectiveness of the proposed features. A test rig as shown in Figure 4 is used to collect fault signals of the rolling bearing. The type of rolling bearing used in the experiment is 6308, with 8 rolling elements. Vibration signals are obtained by an acceleration transducer installed on the bearing seat, with a speed of $1050 \mathrm{r} / \mathrm{min}$ and a sampling frequency of $10240 \mathrm{~Hz}$. There are four types of rolling bearing states, namely, rolling element fault, inner ring fault, outer ring fault, and normal state. Twenty samples are collected for each type of state, hence 80 sets of data in total.

The experiment data of the rolling bearing are processed with the FCN subnetwork average degree extraction method. Firstly, the energy spectrum of the frequency band is established based on time series. To keep the periodic information of the data as much as possible, the frequency bandwidth is chosen as an integral multiple of the bearing rotation frequency or the rotation frequency. The frequency bandwidth $m$ used in this paper is 4 times the rotation frequency. The rotation speed of the bearing during the experiment is $1050 \mathrm{r} / \mathrm{min}$, and the frequency bandwidth is determined as $70 \mathrm{~Hz}$ after calculation.

Based on the energy spectrum of frequency band, a frequency complex network (FCN) model is constructed with the application of recursive algorithm. The adjacency matrix corresponding to the complex network model is the same as recursive matrix. Then, the network is visualized, and the model displayed is a complex network model, unweighted and undirected. The display layout is set as FR (Fruchterman-Reingold); the size of the network nodes is determined by the degree of the nodes. The larger the value of the degree, the larger the size of the nodes and the darker the color. The frequency complex network (FCN) model corresponding to different states of the rolling bearing is shown in Figure 5.

It can be seen from Figure 5 that the complex network shape corresponding to rolling element fault and inner ring fault is similar, but there is still a big difference in the attributes of the nodes and edges. Outer ring faults correspond to complex network with sparse edges and prominent nodes. The normal corresponding complex network is close to a fully connected network. Therefore, different network models under different states of the rolling bearing have different network structures, which lays a good foundation for the extraction of network topological features. 


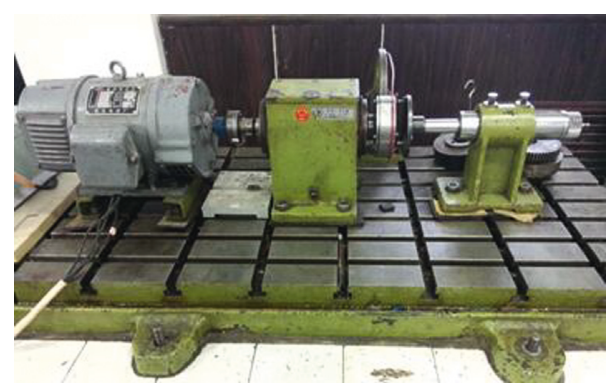

FIgURE 4: Test rig for rolling bearing.

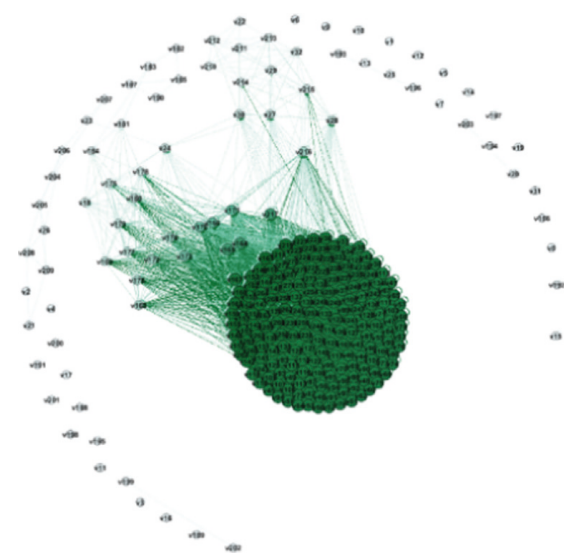

(a)

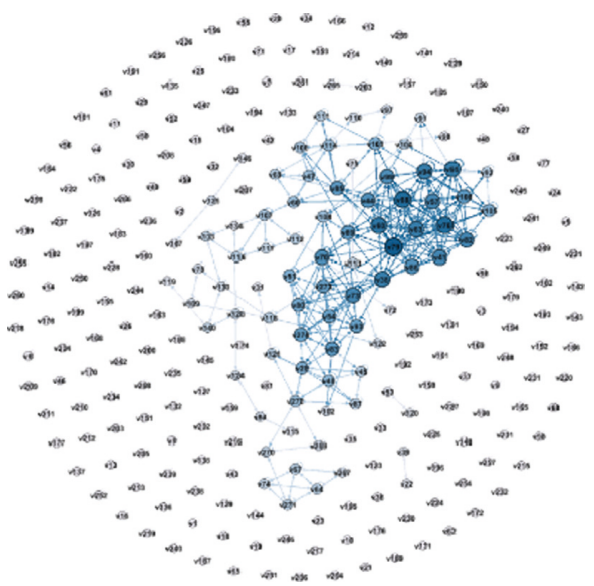

(c)

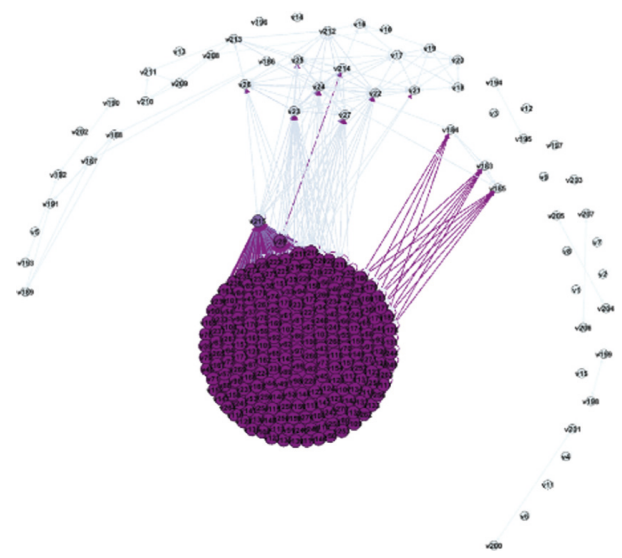

(b)

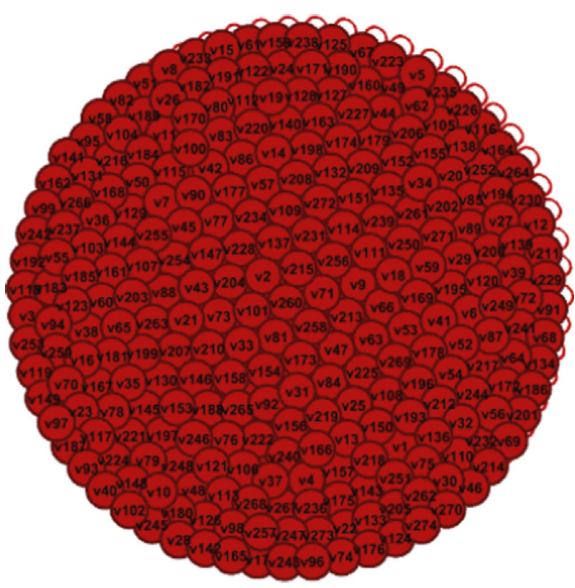

(d)

FiguRE 5: FCN model of rolling bearing's different states. (a) FCN model of rolling element fault. (b) FCN model of inner ring fault. (c) FCN model of outer ring fault. (d) FCN model of normal state.

The FCN model of the rolling bearing in different states is evenly decomposed into 8 subnetworks, and the average degree of nodes in each subnetwork is extracted to form an eight-dimensional feature vector, and the feature distribution diagram of different faults of the rolling bearing is obtained as shown in Figure 6. Wherein, Figure 6(a) is a three-dimensional graph of degree distribution of subnetwork nodes. There are in total 80 samples, and 8-dimensional features are extracted from each sample. Figure 6(b) is a platform of feature distribution, with different colors representing the amplitudes of sample features.
The horizontal axis represents features, and the vertical axis represents samples, with 1-20 groups being rolling element faults, $21-40$ groups being inner ring faults, $41-60$ groups being outer ring faults, and 61-80 being normal.

One sample is chosen from each feature set of the rolling element faults, the inner ring faults, the outer ring faults, and the normal state to draw a bar graph, as shown in Figure 7. It is not difficult to find out that the features of four states (the rolling element faults, the inner ring faults, the outer ring faults, and the normal) differ greatly from each other, especially the normal state and the outer ring fault state. 


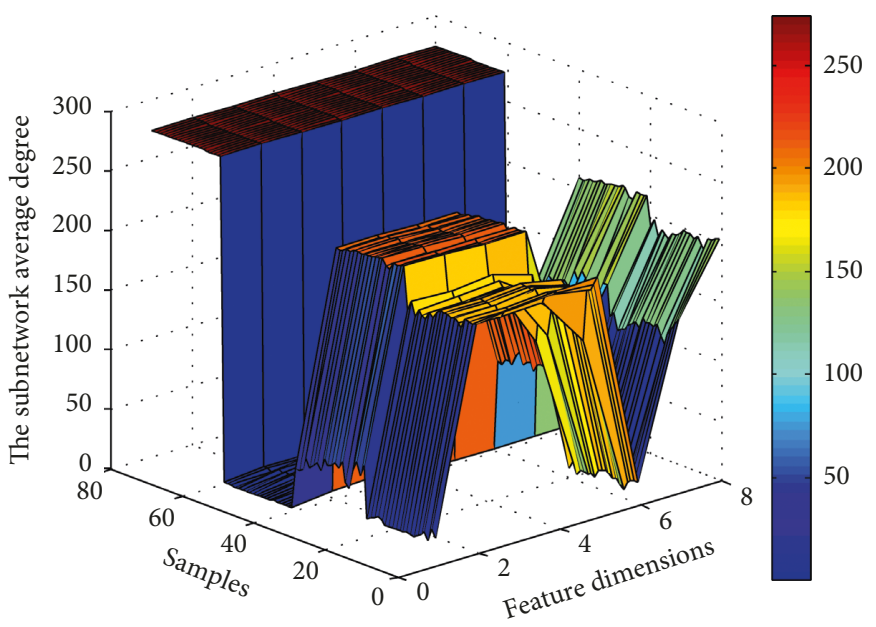

(a)

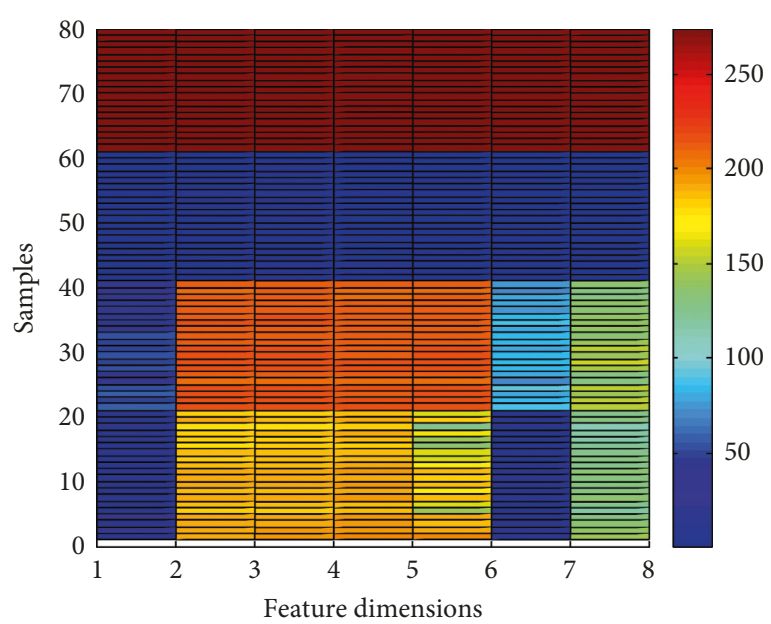

(b)

Figure 6: Different fault features' distribution of rolling bearing. (a) Three-dimensional figure of features' distribution. (b) Platform of features' distribution.

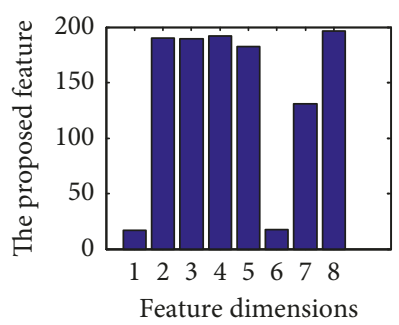

(a)

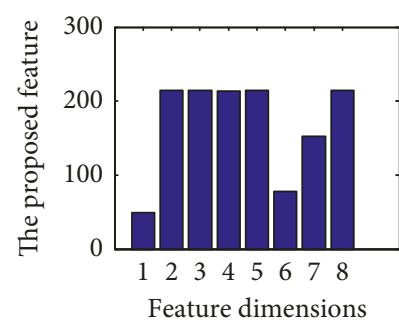

(b)

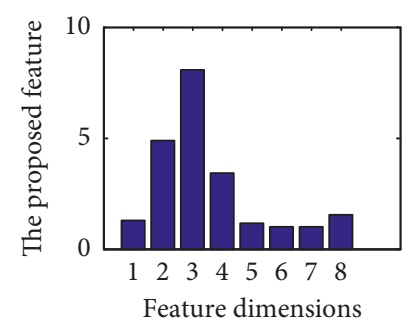

(c)

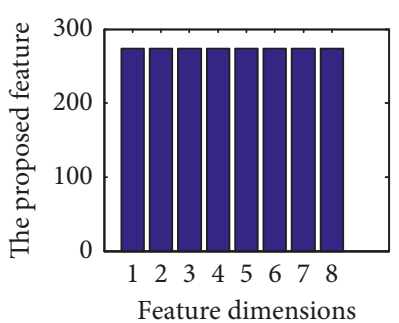

(d)

FiguRE 7: 16D subnetwork average degree. (a) Feature of rolling element fault. (b) Feature of inner ring fault. (c) Feature of outer ring fault. (d) Feature of normal.

For the rolling element faults and the inner ring faults, the features of the $1^{\text {st }}$ and the $6^{\text {th }}$ dimension have great difference.

\subsection{Comparative Analysis}

3.2.1. Feature Separability. The principal component analysis (PCA) algorithm [18] is applied in the dimensional reduction and visualization of average degree features of subnetworks to obtain the feature dimensional reduction graph as shown in Figure 8. It is not difficult to find out that the four samples of the same type of rolling bearing have higher degree of clustering, and there is a clear degree of differentiation with no overlap between different types of samples, which proves that the feature categories proposed in this paper have strong separability. Wherein, the sample clustering degrees of the outer ring fault and the normal sate are the highest and farthest from other sample types, which is consistent with the conclusion drawn from the subnetwork average degree feature diagram above.

The existing complex network methods are mostly modelled based on the time domain, such as limited penetrable visibility graph method [19], and a topological

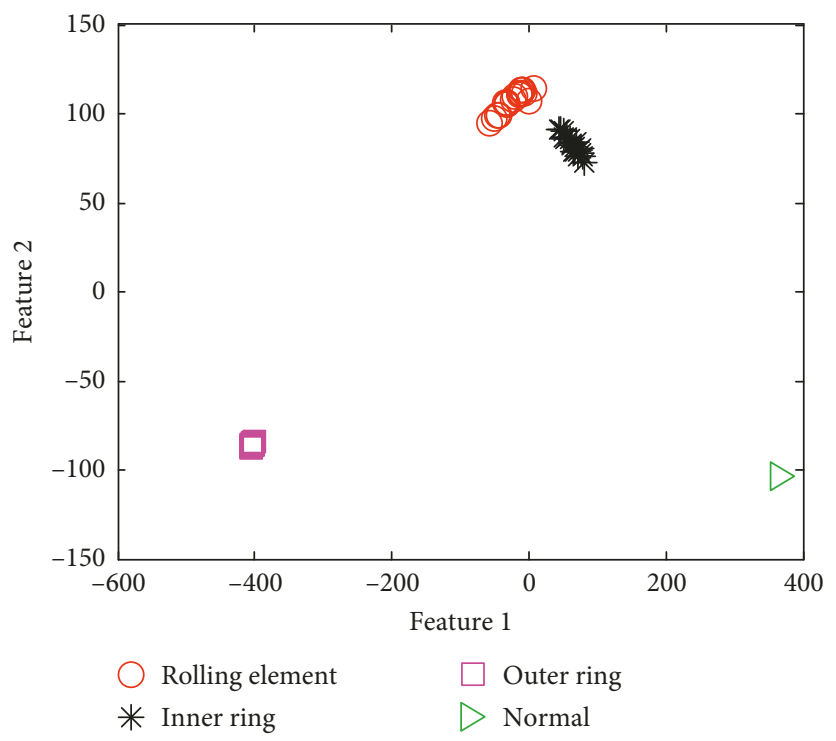

FIgURE 8: Characteristics distribution figure of bearing after PCA.

feature parameter of the network is extracted to represent the entire network. Features such as the average path length, clustering coefficient, and degree, calculated on the basis of 
the entire network, are not sensitive to local changes within the network and hence cannot reflect the microscopic feature of the network. Figure 9 shows the average path length, clustering coefficient, and degree based on limited penetrable visibility graph method of the four states of the rolling bearing, respectively. The horizontal axis represents samples, and there are 20 samples for each state. The vertical axis represents the network topological features corresponding to the samples. It can be seen that the ability of the three features of the average path length, the clustering coefficient, and the degree to distinct different types of samples of rolling bearing is poor. Only the normal state is significantly different from the three faults states which overlap with each other, resulting in difficulty to accurately distinguish with one other. Therefore, compared with the features proposed in this paper, the existing network topological feature categories have poor separability and cannot be applied to subsequent diagnosis and recognition.

The classification separability measure, a distance-based feature evaluation function, is defined as the ratio of interclass divergence and interclass divergence in a sample, which can evaluate the effectiveness of a feature subset. The smaller the value, the better the separability. For the same classifier, the good interclass separability can significantly improve the identification rate of the classifier; and more confounding and overlapping feature information makes pattern recognition very difficult for classifiers. A quantitative comparative analysis is made to network topological feature vector $L C D$, the combination of the average path length $L$, the clustering coefficient $C$, the degree $D$, and subnetwork average degree feature (reduced to three dimensions) proposed in this paper, with the comparison results shown in Table 2 which indicates that the classification separability measure of the proposed features is smaller than that of commonly used features $L C D$. This proves that the features of this paper are superior to the common network topological features and are consistent with the above qualitative analysis results.

3.2.2. Recognition Results. In order to verify the effectiveness of the subnetwork average degree feature in fault diagnosis of rolling bearings, a radial basis function (RBF) neural network classifier [16] is used to conduct the classification test. Ten training samples and 10 testing samples are selected in each state, hence 40 training samples and 40 testing samples in total. For the 40 training samples of the four types, 40 16dimensional feature vectors are generated with the above method. The 40 subnetwork average degree features are trained and classified with RBF neural network, and the different fault classification charts of the rolling bearing are obtained as shown in Figure 10, wherein the first type is the rolling element faults, the second type is the inner ring faults, the third type is the outer ring faults, and the fourth type is the normal state. It is not difficult to find out that when the subnetwork average degree feature is input into the RBF neural network, the recognition accuracy rate can reach $100 \%$.

Time-domain feature parameters, easy to calculate and clear in physical meanings, have been applied in fault detection and state analysis for various types of machinery. In this paper, the following 14 types of time-domain statistical features are applied, namely, mean, standard deviation, variance, skewness, kurtosis, peak-to-peak value, square root amplitude, average amplitude, rms amplitude, extreme value, waveform index, peak index, pulse index, and margin index. The above parameters constitute the feature vectors representing the state of the bearing, which are compared with the features mentioned herein in the RBF recognition accuracy rate. Similarly, 10 training samples are selected for each state, and the remaining 10 groups of samples are identified using the RBF neural network as a classifier, with the recognition results shown in Figure 11. It is not difficult to find out that when the time-domain statistical parameters are input into the RBF neural network, the recognition accuracy rate can reach $97.5 \%$, which indicates that better separability features are very important for subsequent accurate recognition.

In order to more objectively compare the recognition results, 10 groups of samples are randomly selected from each fault set as training samples, and RBF neural network is used as the classifier to identify the remaining 10 groups of samples. The time-domain feature parameters mentioned above and the features in this paper are independently used. The recognition results in Table 3 are the mean and variance of the accuracy rate of each algorithm after running 10 times independently in MATLAB. The results indicate that the mean value of the feature recognition accuracy rate in this paper is high, and the variance is small, which proves that the features in this paper are not only good in classification separability, but also relatively stable, with no significant fluctuation in the recognition accuracy for different training data in the same data set.

3.2.3. Computing Efficiency. Table 4 lists the average value of the operation times of the four feature extraction methods. The experimental computer is configured as Inter (R) Core (TM) i5-3470 CPU $3.2 \mathrm{GHz}$. The test environment is Windows 7. The programming software is MATLAB R2012a. It can be seen from Table 4 that although the proposed method takes longer than the time-domain statistical indicators, it is shorter than the existing network features, and it basically meets the engineering application requirements.

\section{Assessment of Bearing Performance Degradation}

\subsection{Degradation Feature Extraction and State Warning}

4.1.1. Feature Extraction. The life cycle data of rolling bearings published by the University of Cincinnati are applied to validate the method proposed in this paper. Four Rexnord ZA-2115 rolling bearings are installed on the bearing test stand, with a rotational speed of $2000 \mathrm{r} / \mathrm{min}$. A radial load of $26.6 \mathrm{kN}$ is applied to the shaft and the bearing. One PCB353B33 accelerometer is installed in the $X$ and $Y$ directions of each bearing, with a sampling frequency of 


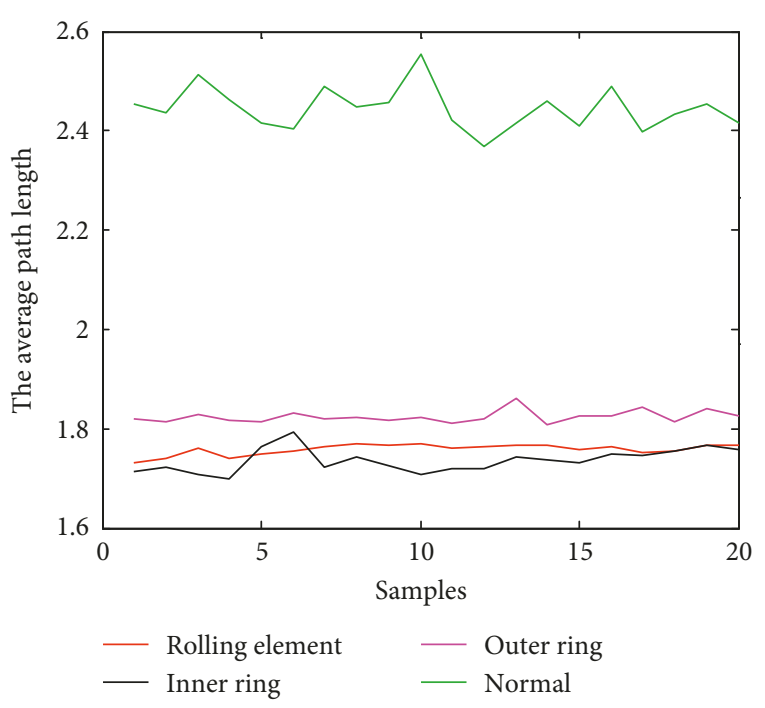

(a)

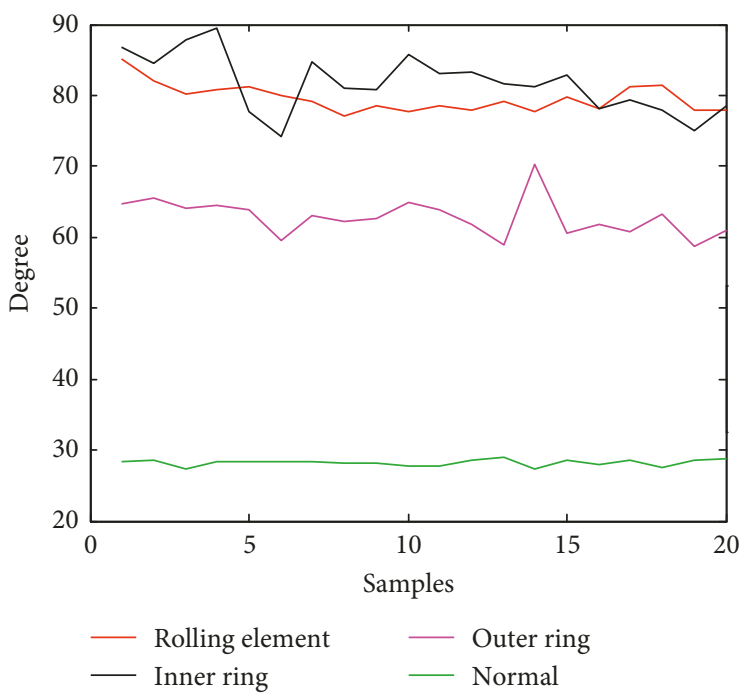

(b)

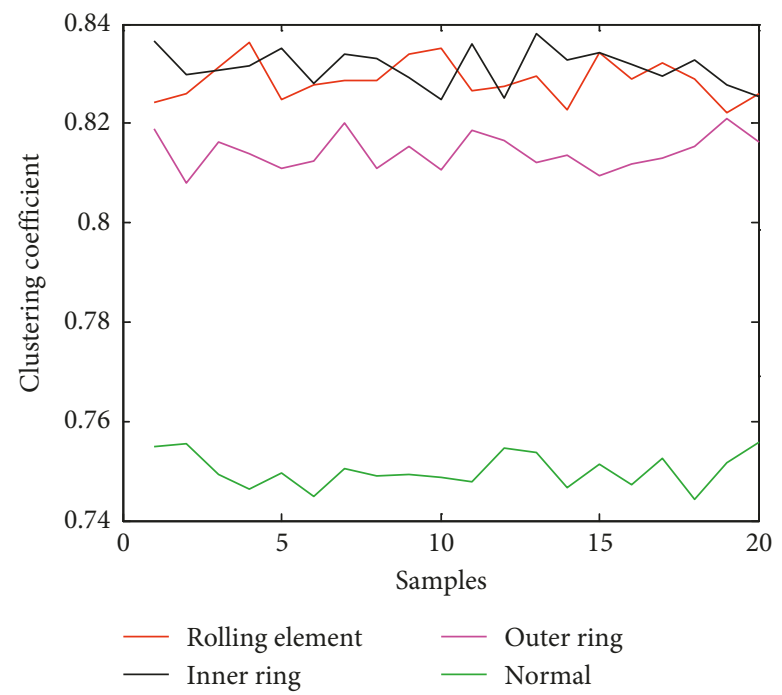

(c)

FIgURE 9: Feature distribution of four bearing states. (a) The average path length. (b) Degree. (c) Clustering coefficient.

$20 \mathrm{kHz}$. The vibration data are collected once every 10 minutes, and the data length is 20480 points. In this paper, the data of bearing 1 in experiment 2 are selected, with a total of 984 samples during the experiment, and bearing 1 is seriously damaged [20].

The method proposed in this paper is applied to extract the performance degradation index. The corresponding band energy spectrum of each sample is obtained. In order to retain the periodic information of the data as much as possible, the frequency bandwidth $m$ used in this paper is the bearing rotation frequency. The $500^{\text {th }}, 533^{\text {rd }}, 600^{\text {th }}, 702^{\text {nd }}$, $823^{\text {rd }}$, and $950^{\text {th }}$ samples are successively selected to draw their band energy spectrum, as shown in Figure 12. It is clear that the spectral peaks of the energy spectrum in the fault frequency band are more and more prominent with the deepening of the fault degree. Since the intervals between the spectral peaks are the same, the self-similar features of the energy spectrum are more and more obvious.
The frequency band energy spectrum is regarded as a one-dimensional sequence, and a recursive algorithm is applied to extract the self-similar features of the sequence. By setting the delay time as $\tau=6$ and embedding dimension as $s=2$, a recursive matrix corresponding to the energy spectrum sequence can be obtained. Donner et al. found that the recursive matrix can be regarded directly as the adjacency matrix of the network, the sequence is described in the form of a network, and self-similar feature inside the sequence is reflected by the structural features of the network. A recursive algorithm is applied to transform each sample into a complex network model represented by points and edges. The average degree features of each network model are then extracted. The average degree change curve of the entire experimental sample is shown in Figure 13, and the first half of the curve is comparatively steady, which means that the bearing is in normal state. The curve suddenly drops at the $533^{\text {rd }}$ point, and then it comes into a more stable 
TABLE 2: Sort separability criterion of different features.

\begin{tabular}{lcc}
\hline Feature & LCD & $\begin{array}{c}\text { The proposed } \\
\text { feature }\end{array}$ \\
\hline $\begin{array}{l}\text { The classification separability } \\
\text { measure }\end{array}$ & 0.1029 & 0.0293 \\
\hline
\end{tabular}
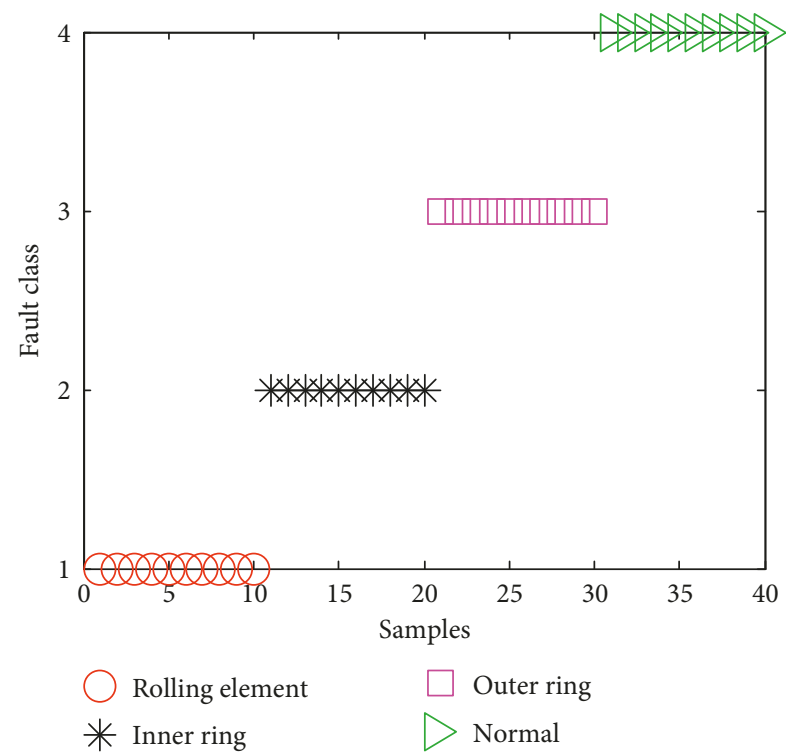

Figure 10: Classification result of bearing.

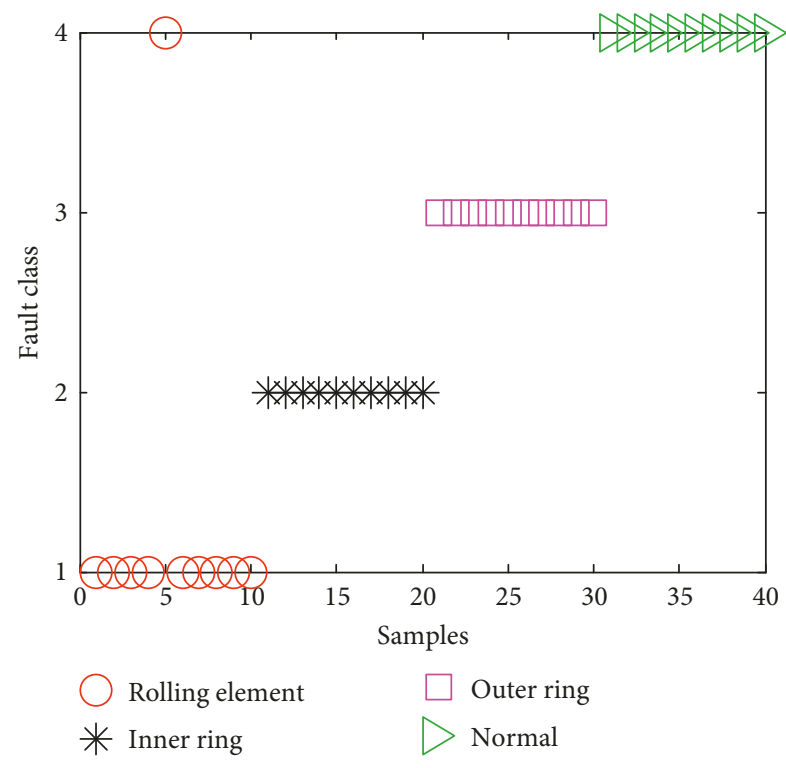

FIgURE 11: Classification result of bearing.

decline phase, which means that the bearing is experiencing early weak faults, and the fault degree gradually deepens. The curve drops sharply at the $702^{\text {nd }}$ point and then slowly increases, indicating that the bearing may have a partial fault and spall. The sharp part of the bearing is gradually flattened. The curve falls sharply again at the $823^{\text {rd }}$ point and became more and more unstable, indicating that the bearing is in
TABLE 3: RBF recognition results based on different features.

\begin{tabular}{lccc}
\hline Feature & & $\begin{array}{c}\text { Time-domain } \\
\text { statistics }\end{array}$ & The proposed feature \\
\hline \multirow{2}{*}{ Accuracy $a(\%)$} & Mean & 98.75 & 100 \\
& Variance & 0.031 & 0 \\
\hline
\end{tabular}

TABle 4: Average running times of different feature extraction methods.

\begin{tabular}{lc}
\hline Feature extraction methods & Time $(\mathrm{s})$ \\
\hline The average path length & 0.9755 \\
Degree & 0.8020 \\
Clustering coefficient & 0.8183 \\
Time-domain statistics & 0.0656 \\
The proposed feature & 0.8027 \\
\hline
\end{tabular}

a state of serious degradation until it completely fails. It is not difficult to find out that the average degree feature is sensitive to the early faults of the rolling bearing. With the degradation of the bearing, the feature value gradually decreases and the fluctuation amplitude gradually increases.

4.1.2. State Monitoring and Warning. The above analysis indicates that the average degree feature tends to gradually decrease with the performance degradation of the rolling bearing. Therefore, a threshold can be set, and when the average degree exceeds the threshold, a fault alarm is given. The threshold is determined with the Layda rule in this paper. When the absolute value of the difference between the data point of the average degree and the mathematical expectation of the set is greater than 3 standard deviations, that is $\left|x_{i}-\bar{x}\right|>3 \sigma$, the data point is identified as abnormal. In order to avoid accidental errors, the situation that abnormality occurs to the average degree in five consecutive times is defined as device abnormal, and thereby a fault alarm is given. As shown in Figure 14, an alarm of abnormal data is given in the $533^{\text {rd }}$ set of data with the method proposed in this paper, indicating that the average degree has exceeded the threshold range five times successively, which proves that this method is sensitive to the early fault of the bearing and can detect the exact time of the early fault, so as to succeed in the monitoring and early warning of the bearing.

4.1.3. Comparative Analysis. Time-domain parameters, statistically easy to calculate and clear in physical meanings, have long been applied in state monitoring and fault diagnosis of rolling bearings, among which mean square value $C$, kurtosis $K$, etc., are often used as index of bearing performance degradation [21]. The mean square value and kurtosis curves of the entire experimental data are shown in Figures 15 and 16. With the deterioration of bearing faults, the mean square value and kurtosis tend to increase, but both begin to show a significant increase at the $702^{\text {nd }}$ sample, and early faults could not be detected. In addition, the kurtosis fluctuates in the normal stage and has poor stability. 


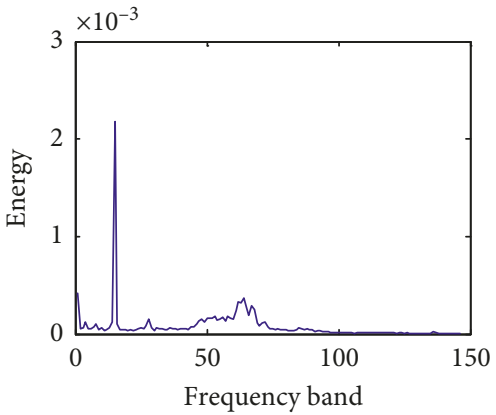

(a)

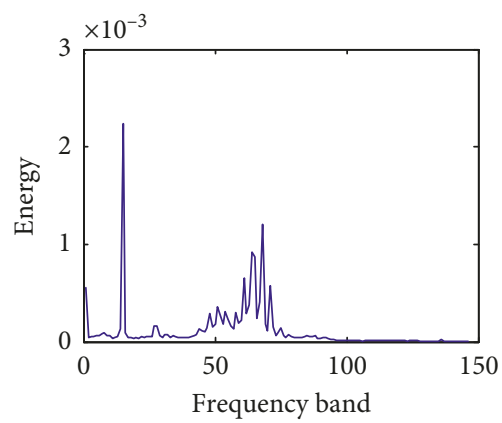

(d)

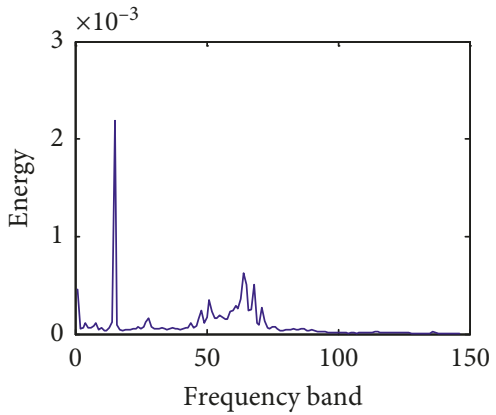

(b)

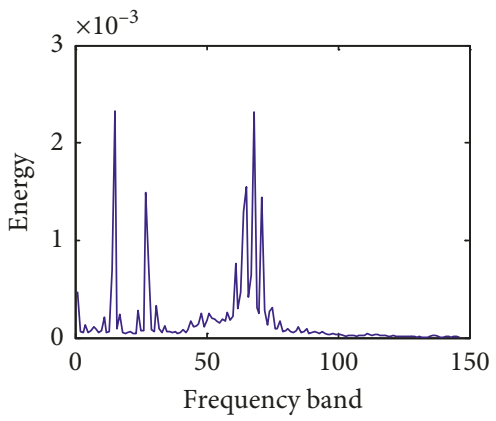

(e)

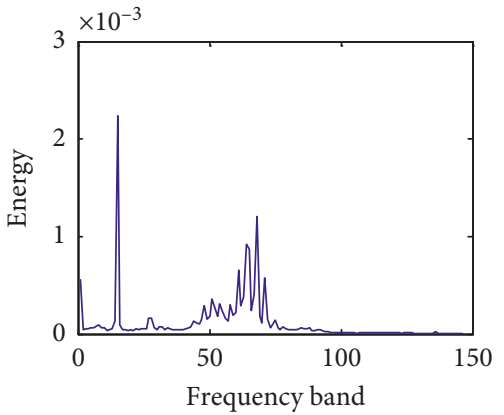

(c)

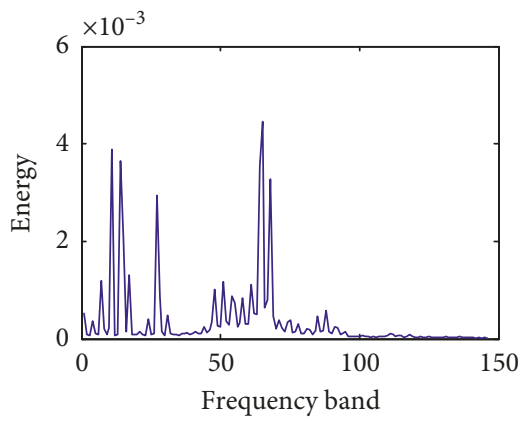

(f)

Figure 12: Frequency band energy spectrum of some samples. (a) The $500^{\text {th }}$ sample. (b) The $533^{\text {rd }}$ sample. (c) The $600^{\text {th }}$ sample. (d) The $702^{\text {nd }}$ sample. (e) The $823^{\text {rd }}$ sample. (f) The $950^{\text {th }}$ sample.

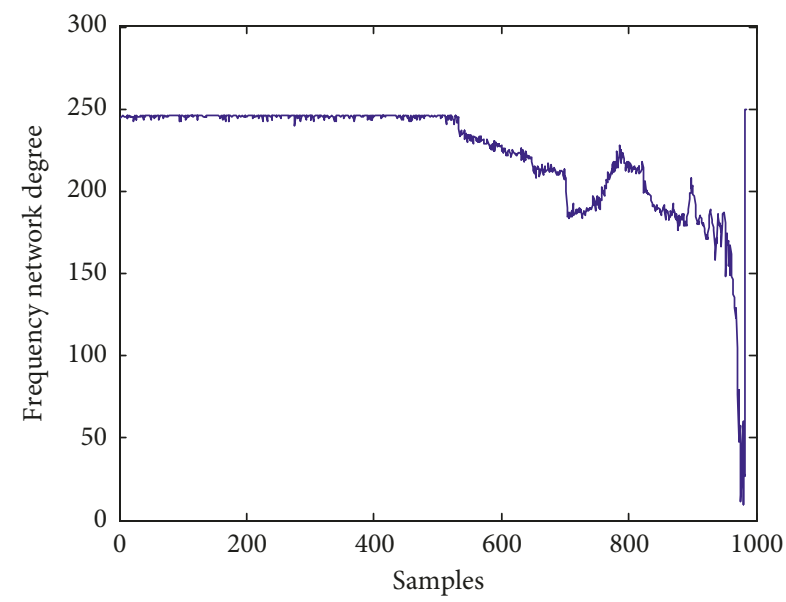

Figure 13: The average degree of bearing all life.

Therefore, the network average degree index is better in characterizing bearing performance degradation than the traditional time-domain statistical parameters, namely, mean square value and kurtosis, and hence early bearing faults of bearings can be identified and correct early warning can be given.

\subsection{Degradation State Recognition}

4.2.1. Degradation State Recognition Based on Subnetwork Average Degree. On the basis of the average degree feature of the bearing life, the complex network model is evenly

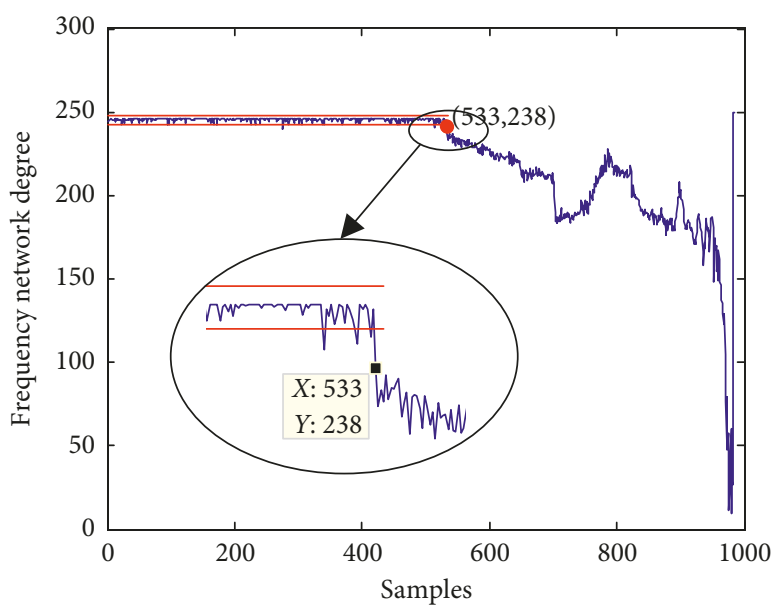

FIgURE 14: Bearing performance degradation alarm line.

decomposed into 16 subnetworks. The average degree of nodes in each subnetwork is extracted to form the multidimensional degradation feature vector to identify the degradation state of the bearing, that is, the average degree of the subnetwork, $\vec{p}=\left[p_{1}, p_{2}, p_{3}, \ldots, p_{16}\right]$. The average degree change curve of the subnetwork for the entire experimental samples is shown in Figure 17. Since the feature vector is composed of the degree of nodes in different areas of the network, there is no redundant information between the values. Hence, it is not necessary to perform dimensionality reduction on the feature vector. As can be seen from Figure 18, the change of each value in the feature vector is consistent with 


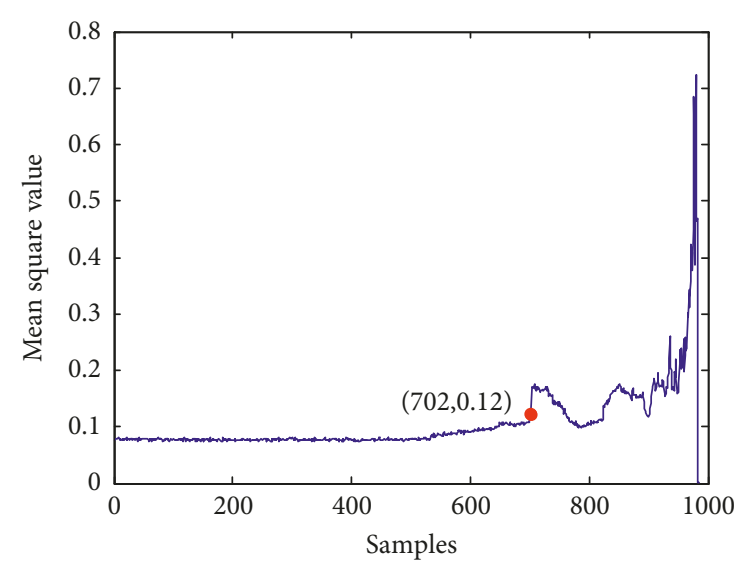

FIgURE 15: Root mean square value of bearing all life.

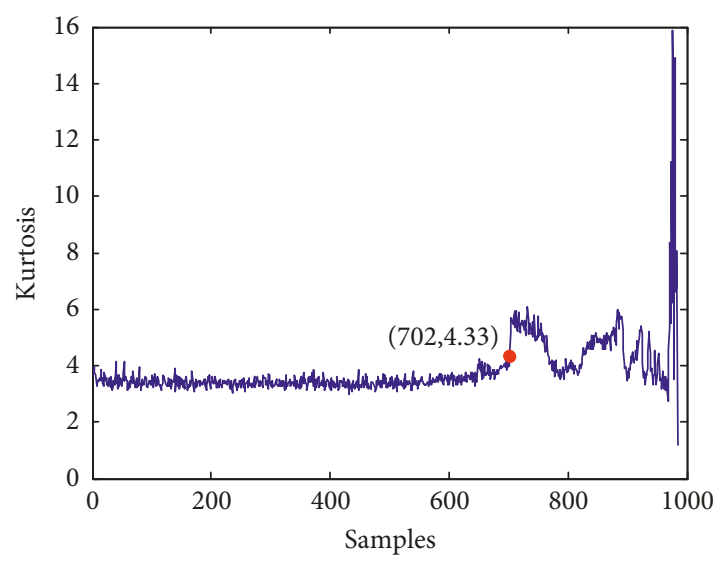

Figure 16: Kurtosis of bearing all life.

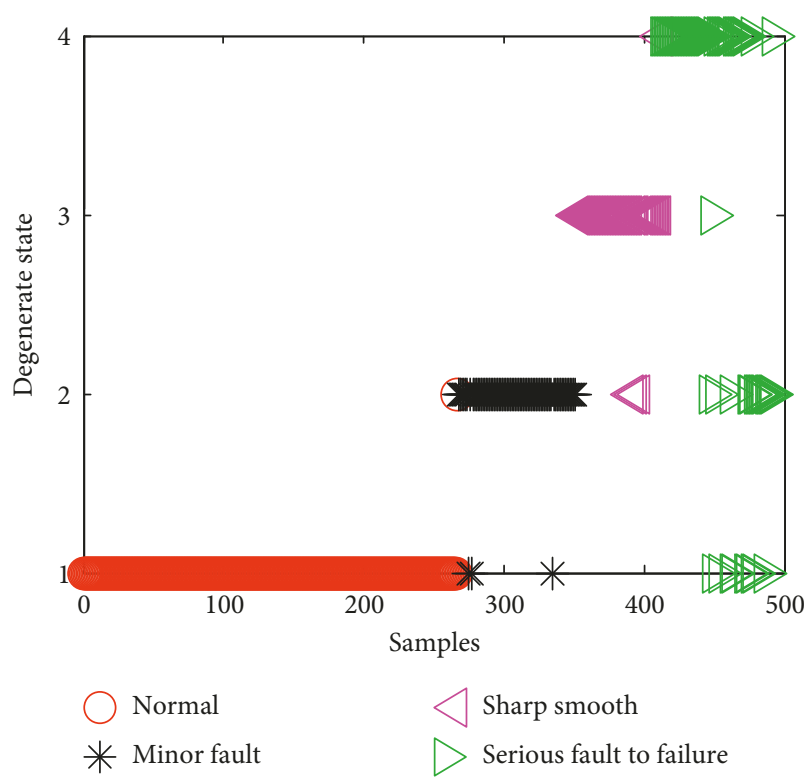

FIgURE 17: The recognition result of bearing degenerative state.

the development trend of the fault, with some values sensitive to the fault and some value relatively stable, which can effectively describe the whole life process of the bearing.

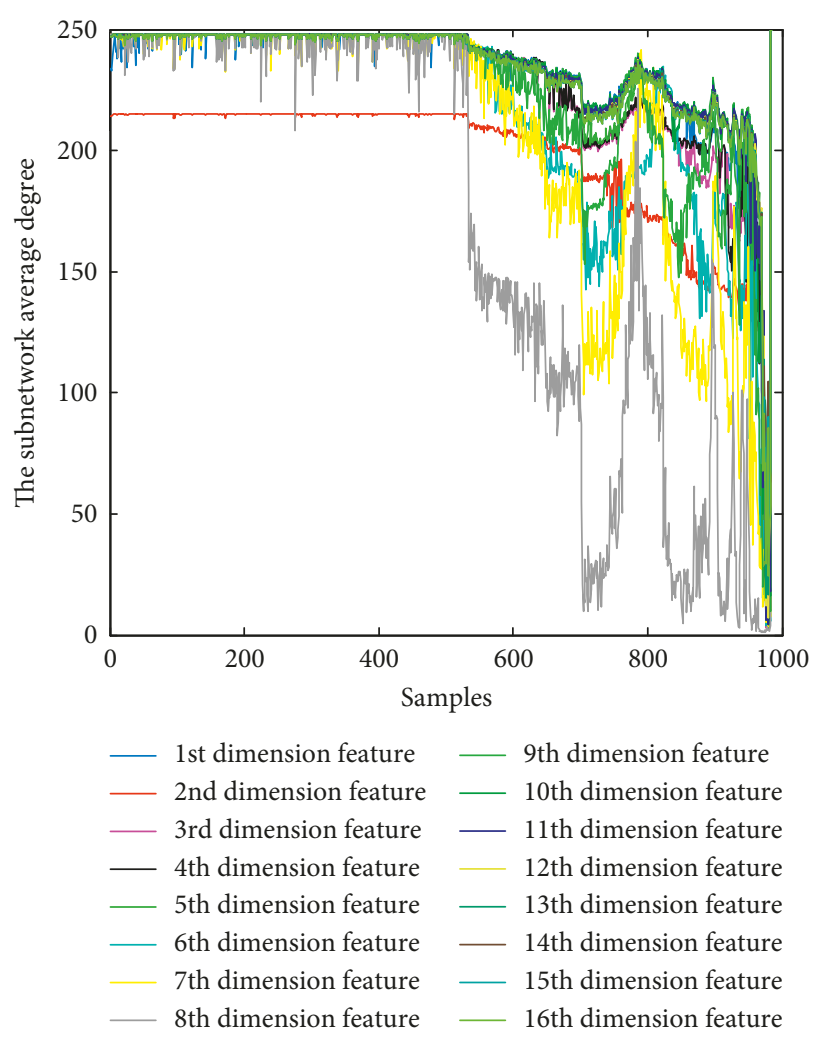

FIGURE 18: Subnetwork average degree of bearing all life.

In order to further understand the subnetwork average degree feature vectors, principal component analysis (PCA) algorithm is applied to reduce the dimension of the subnetwork average degree feature to obtain the feature reduction dimension shown in Figure 19. In the four degradation states of the rolling bearing, the similar samples have higher degree of aggregation, and there is only partial overlapping between the different types of samples, which indicates that the multidimensional degradation feature vectors mentioned in this paper can meet the requirements for distinguishability of different states. Wherein, the normal state has the highest degree of aggregation, and the serious degradation to the fault state has the lowest degree, which accords with the basic features of the degradation of the rolling bearing.

The radial basis function (RBF) neural network classifier is applied to identify the four states of the rolling bearing life cycle. The first stage of the bearing is the normal state and contains the first to $533^{\text {rd }}$ samples; the second stage is the minor fault, including the $534^{\text {th }}$ to $702^{\text {nd }}$ samples; the third stage is deepening of the fault and gradual thinning of the sharp part, including the $703^{\text {rd }}$ to $823^{\text {rd }}$ samples; the fourth stage is serious fault to failure, including the $824^{\text {th }}$ to $984^{\text {th }}$ samples. The samples in each stage are divided into two sample sets according to the parity of the sequence, with one sample set selected as the training sample set, and the other set is selected as the testing sample set. The training sample feature vector set $V_{\mathrm{T}}$ is the training data set of RBF neural network, among which there are 267 training samples in the 


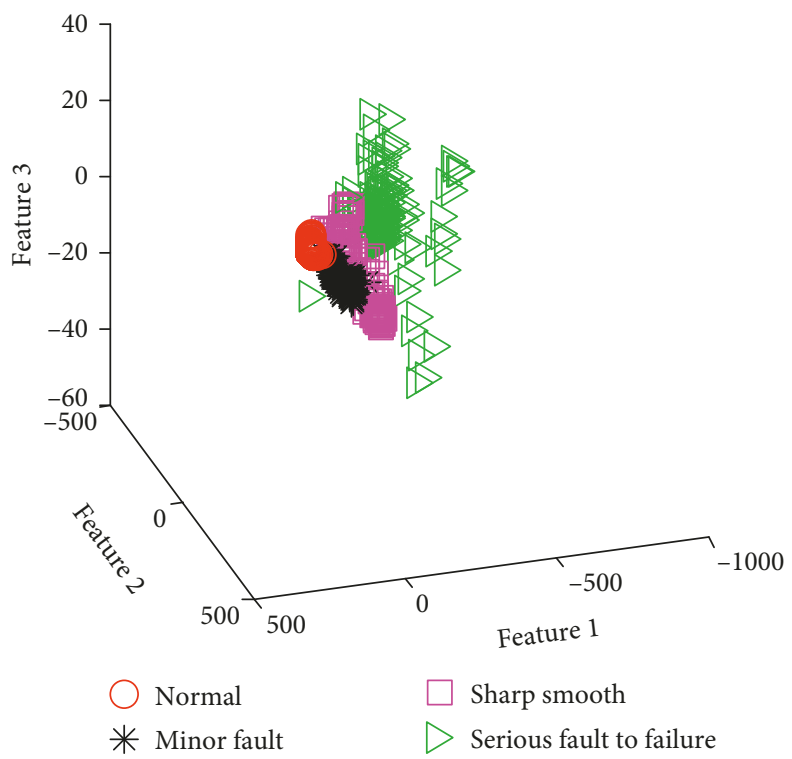

FIGURE 19: Dimension reduction results of subnetwork average degree.

TABLE 5: RBF recognition results based on different features.

\begin{tabular}{lccc}
\hline Feature & Time-domain statistics & Frequency-domain statistics & The proposed feature \\
\hline Accuracy (\%) & 59.55 & 70.33 & 93.09 \\
\hline
\end{tabular}

first stage, 84 training samples in the second stage, 61 training samples in the third stage, and 80 training samples in the fourth stage. Then, the network is tested and verified with the feature vector set $V_{\mathrm{C}}$ of the testing sample. The degradation state of the testing sample is identified, and the result of the degradation recognition of the rolling bearing is obtained as shown in Figure 17, with a recognition accuracy rate of $93.09 \%$.

It can be seen from Figure 17 that the first three states of the rolling bearing life cycle have high recognition accuracy, while for the serious fault to failure state, a large number of samples are identified as other states, and the recognition accuracy is low. The reason may lie in the unstable performance during the serious fault state, resulting in the fluctuation of the extracted features and the low similarity between samples. However, the entire recognition results can basically meet the requirements of the state recognition of bearing degradation.

4.2.2. Comparative Analysis. Statistical parameter diagnosis, a relatively effective diagnostic method, has been applied since a long time ago, with the time-domain statistical parameter the most commonly used one. For rolling bearings, the frequency components of the signal, the energy of different frequency components, and the peak position of the main frequency spectrum of the energy spectrum will also change when a fault occurs. Therefore, the statistical parameters in the frequency domain are often applied to identify the degradation stats of the bearing. In this paper, 14 kinds of time-domain feature parameters [20] and 12 kinds of frequency-domain feature parameters [22] are used to characterize the bearing state and are compared with the features proposed in this paper in RBF recognition rate of bearing degradation. Similarly, the feature vector set $V_{\mathrm{T}}$ of the training sample is used as the training data set of the RBF neural network, and then the network is tested and verified with the feature vector set $V_{\mathrm{C}}$ of the testing sample to identify the degradation state of the testing sample, with the recognition results shown in Table 5. Compared with the time-domain feature parameters and the frequency-domain feature parameters, the average degree of the subnetworks proposed in this paper is more accurate in identifying the degradation state of the bearings.

\section{Conclusion}

In order to overcome the deficiencies of existing complex network modelling methods and conventional network topological features, this paper constructs a new complex network model from the perspective of frequency domain and makes application of complex network decomposition to extract the average degree characteristics of subnetworks sensitive to local changes in the network based on the average degree of the bearing condition monitoring and early warning method and the average degree of the subnetwork bearing degradation state recognition method, which are applied in the life cycle of the rolling bearing detection. The results show that the mean and variance of the recognition accuracy of the proposed method are better than those of the time-domain statistics. The average degree of structural features of complex network can effectively characterize the 
degradation process of bearings and is more sensitive to early weaker faults than commonly used time-domain statistical parameters such as kurtosis and RMS values. The degradation recognition method based on average degree of subnetworks can effectively identify the degradation state of bearings, with a recognition rate of $93 \%$, which is higher than the time-domain statistical parameter and the frequency-domain statistical parameter. The method and the degradation features extracted in this paper are sensitive to the early faults and have high accuracy in degradation recognition, which can meet the requirements of the degradation assessment of rolling bearing performance.

\section{Data Availability}

The data used to support the findings of this study are available from the corresponding author upon request.

\section{Conflicts of Interest}

The authors declare that they have no conflicts of interest.

\section{Acknowledgments}

This study was supported by the National Natural Science Foundation (51775409), Equipment Pre-Research Sharing Technology and Domain Funds (6140004030116JW08001), and National Key Research and Development Project (2017YFF0210504).

\section{References}

[1] S. Wang, W. Huang, and Z. K. Zhu, "Transient modeling and parameter recognition based on wavelet and correlation filtering for rotating machine fault diagnosis," Mechanical Systems and Signal Processing, vol. 25, no. 4, pp. 1299-1320, 2011.

[2] J. Luo and Y. Zhong, "Research and application of wavelet packet time-frequency analysis," Journal of Chongqing University of Posts and Telecommunications (Natural Science Edition), vol. 21, no. 3, pp. 379-387, 2009.

[3] X. Hu, J. Wang, Y. Ji et al., "The application of the wavelet analysis of analytic signals in mechanical fault diagnosis of circuit breakers," in Proceedings of IEEE Instrumentation and Measurement Technology Conference, vol. 2, pp. 1477-1482, Vail, CO, USA, May 2003.

[4] J. Zhang and M. Small, "Complex network from pseudoperiodic time series: topology versus dynamics," Physical review letters, vol. 96, no. 23, article 238701, 2006.

[5] X. Xu, J. Zhang, and M. Small, "Superfamily phenomena and motifs of networks induced from time series," Proceedings of the National Academy of Sciences, vol. 105, no. 50, pp. 19601-19605, 2008.

[6] J. Fang, X. Wang, and Z. Liu, "Research on the complexity problem and nonlinear complex network system," Science and Technology Review, vol. 22, no. 2, pp. 9-12, 2004, in Chinese.

[7] J. Zhang, J. Sun, X. Luo, K. Zhang, T. Nakamura, and M. Small, "Characterizing pseudoperiodic time series through the complex network approach," Physica D: Nonlinear Phenomena, vol. 237, no. 22, pp. 2856-2865, 2008.

[8] L. Lacasa and R. Toral, "Description of stochastic and chaotic series using visibility graphs," Physical Review E Statistical
Nonlinear and Soft Matter Physics, vol. 82, no. 2, article 036120, 2010.

[9] Z. Ming, W. Er-Hong, Z. Ming-Yuan, and M. Qing-Hao, "Directed weighted complex networks based on time series symbolic pattern representation," Acta Physica Sinica, vol. 66, article 210502, 2017.

[10] B. Sun, C. Liang, and D. Shang, "Rolling bearing fault diagnosis based on recursive complex network," Journal of Vibration, Measurement and Diagnosis, vol. 35, no. 3, pp. 578-584, 2015.

[11] J. P. Eckmann, S. O. Kamphorst, and D. Ruelle, "Recurrence plots of dynamical systems," Europhysics Letters, vol. 4, no. 9, pp. 973-977, 1987.

[12] X. Wang, Complex Network Theory and Its Application, Tsinghua University Press, Beijing, China, 2006.

[13] L. Xiang, G. Tang, and A. Hu, "Vibration signal's time frequency analysis and comparison for a rotating machinery," Journal of Vibration and Shock, vol. 29, no. 2, pp. 42-45, 2010.

[14] C. Liu and H. Zhao, "ECOG classification based on frequency band normalization and SVM-RFEd," Chinese Journal of Scientific Instrument, vol. 32, no. 3, pp. 534-539, 2011.

[15] J. E. Meng, S. Wu, J. Lu, and H. L. Toh, "Face recognition with radial basis function (RBF) neural networks," IEEE Transactions on Neural Networks and Learning Systems, vol. 13, no. 3, pp. 697-710, 2002.

[16] F. Cong, J. Chen, G. Dong, and F. Zhao, "Short-time matrix series based singular value decomposition for rolling bearing fault diagnosis," Mechanical Systems and Signal Processing, vol. 34, no. 1-2, pp. 218-230, 2013.

[17] X. L. Yao, H. L. Chen, X. Zhao et al., "Weak link determination of anti-shock performance of shipboard equipment based on paula criterion," Chinese Journal of Ship Research, vol. 2, no. 5, pp. 10-14, 2007.

[18] W. Stacklies, H. Redestig, M. Scholz, D. Walther, and J. Selbig, "pcaMethods-a bioconductor package providing PCA methods for incomplete data," Bioinformatics, vol. 23, no. 9, pp. 1164-1167, 2007.

[19] H. Cheng-Yu, M. Xiao-Fei, and N. Xin-Bao, "Research of short-term heart rate variability during sleep based on limited penetrable horizontal visibility graph," Acta Physica Sinica, vol. 66, article 160502, 2017.

[20] H. Qiu, J. Lee, J. Lin, and G. Yu, "Wavelet filter-based weak signature detection method and its application on rolling element bearing prognostics," Journal of Sound and Vibration, vol. 289, no. 4-5, pp. 1066-1090, 2006.

[21] W. Li, A. Xu, J. Sun, and F. Fan, "Research of wavelet neural network state degradation prediction of rolling bearing new time domain index," Journal of Mechanical Transmission, vol. 40, no. 6, pp. 36-41, 2016.

[22] Y. Lei, Z. J. He, and Y. Y. Zi, "Fault diagnosis based on novel hybrid intelligent model," Chinese Journal of Mechanical Engineering, vol. 44, no. 7, pp. 112-117, 2008. 


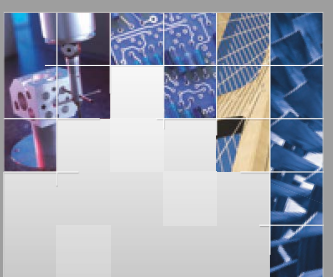

\section{Enfincering}
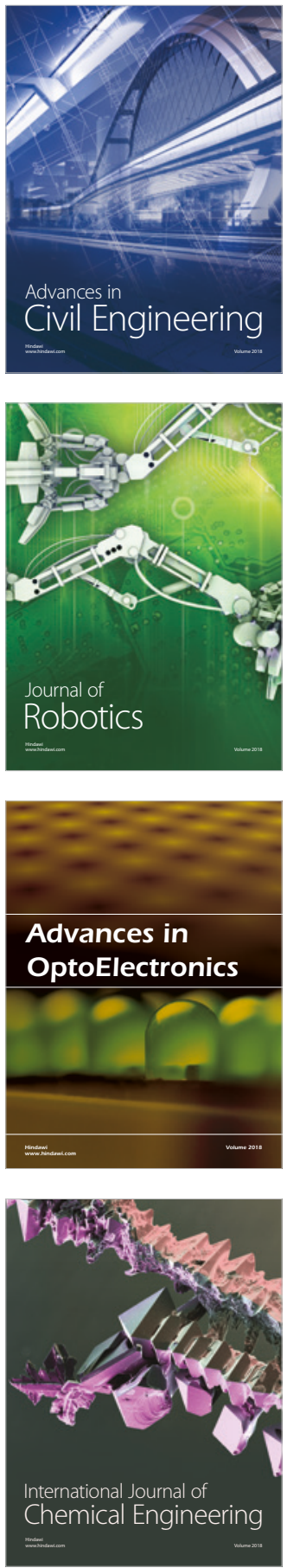

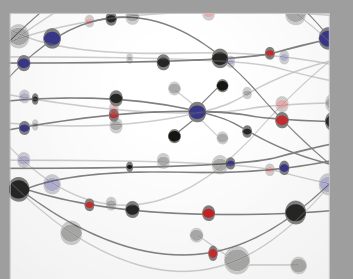

\section{Rotating \\ Machinery}

The Scientific World Journal

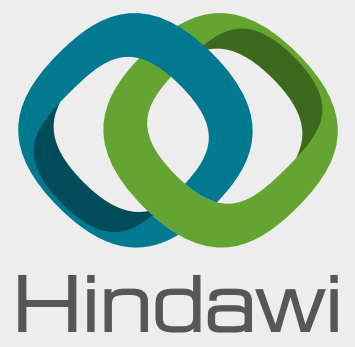

Submit your manuscripts at

www.hindawi.com
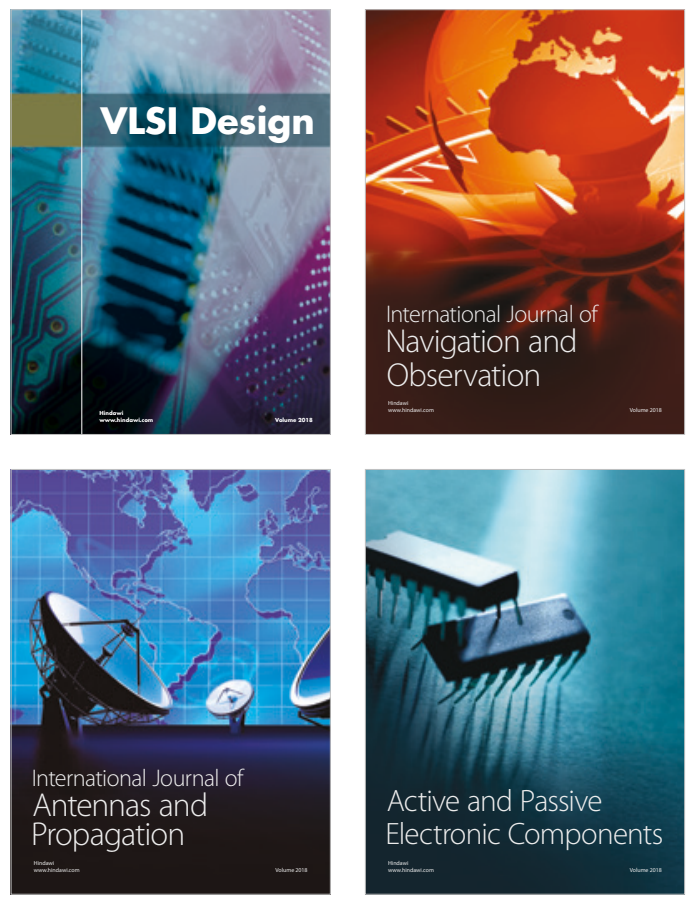
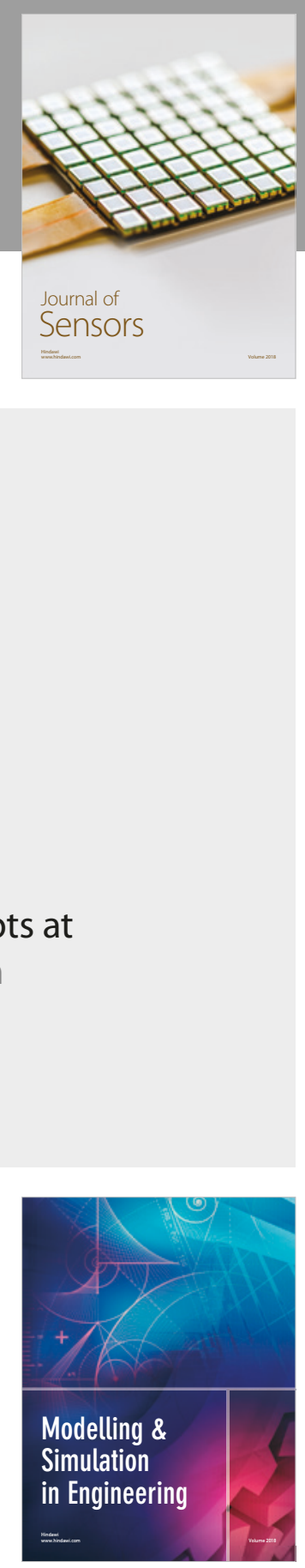

\section{Advances \\ Multimedia}
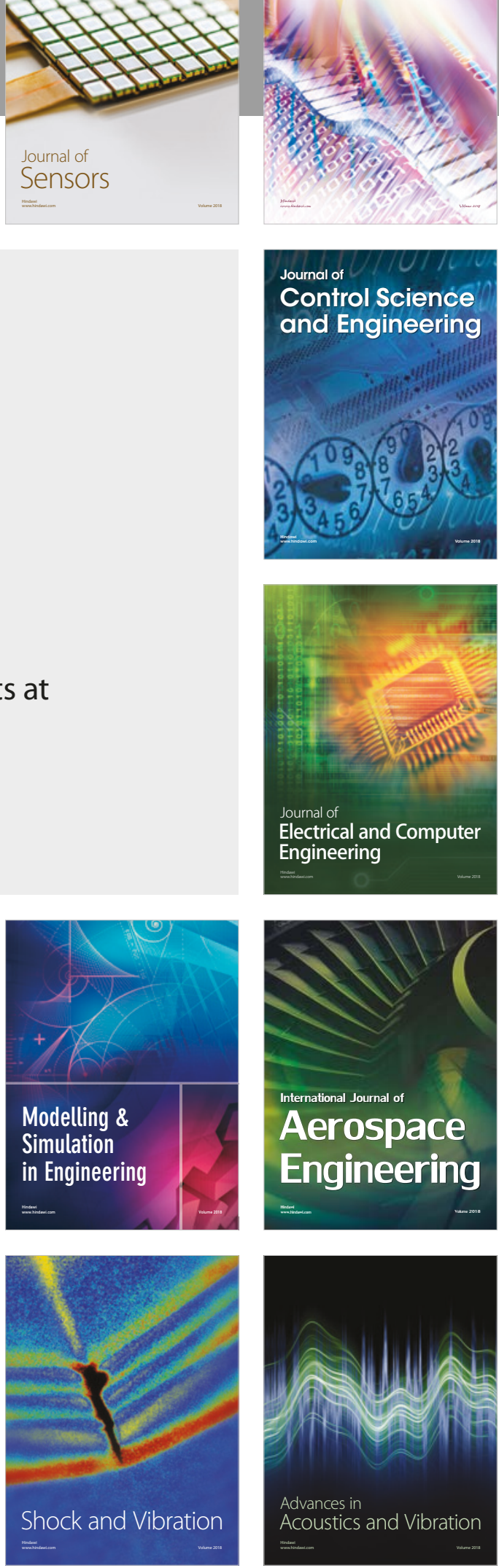